Supplementary Information for:

\title{
Complex System Assembly Underlies a Two-Tiered Model of Highly Delocalized Electrons
}

\author{
Miguel Mompeán ${ }^{1, *}$, Aurora Nogales ${ }^{2}$, Tiberio A. Ezquerra ${ }^{2}$ and Douglas V. Laurents ${ }^{1, *}$ \\ ${ }^{1}$ Instituto de Química Física "Rocasolano" (IQFR-CSIC), Serrano 119, Madrid E-28006, Spain. \\ ${ }^{2}$ Instituto de Estructura de la Materia (IEM-CSIC), Serrano 121, Madrid E-28006, Spain. \\ Correspondence: mmompean@iqfr.csic.es, dlaurents@iqfr.csic.es
}




\section{CONTENTS:}

-Supplementary Figures

-Figure S1: Interaction energies in GNNQQNY and GGGGGGG.

-Figure S2: Effective number of hydrogen bonds.

-Figure S3: Interaction energies with and without van der Waals corrections in MVGGVV and GNNQQNY

-Figure S4: Schematic representation of the experimental procedures.

-Figure S5: Molecular structures of DMSO and DMSO-d6.

-Figure S6: Geometries of the peptides used in the calculations and experiments.

\section{-Supplementary Tables}

-Table S1: NBO analysis of the HBC.

-Table S2: NBO analysis of the hydrogen bonds. 


\section{-Supplementary Figures}

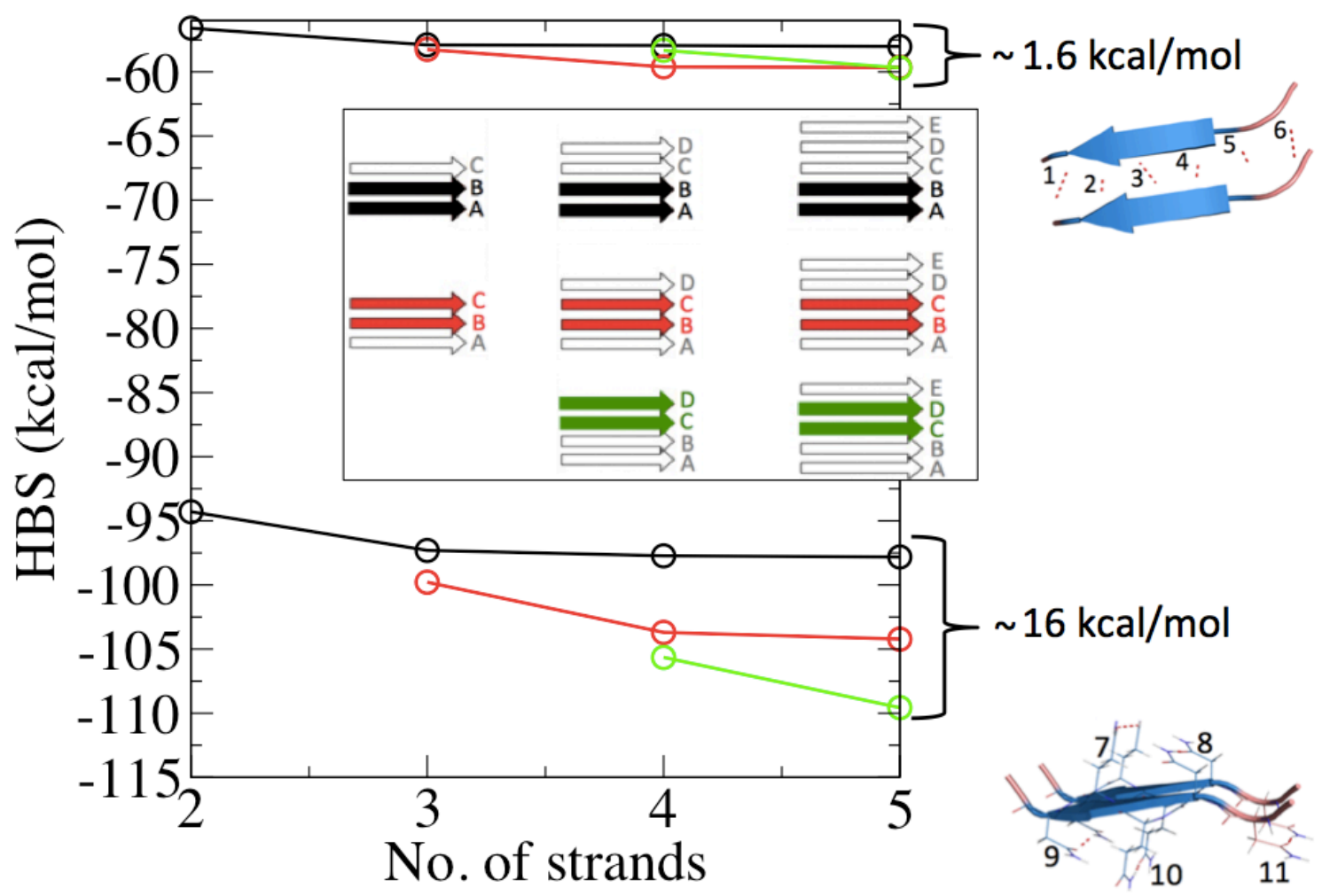

Supplementary Figure 1. Interaction energies in GNNQQNY and GGGGGGG (polyGly). The calculations shown in Fig. 2 for GNNQQNY were repeated on a system in which all residues are mutated to glycine. The resulting system is a symmetric oligomer lacking side chains. Note that only six backbone-backbone (bb-bb) H-bonds are present. The interaction energies (HBS) are stronger for the buried strands with respect to those at the edges and, $\mathrm{A} \cdots \mathrm{BC}$ is similar to $\mathrm{AB} \cdots \mathrm{C}$ due to the monomer symmetry. The marginal change in HBS as the GGGGGGG oligomer grows indicates that hyper-HBC is not present in this system. The same occurs in the pairs $\mathrm{A} \cdots \mathrm{BCD} /$ $A B C \cdots D$ \& $A B C \cdots D E \& A B \cdots C D E$. The inclusion of side chains (experimental systems, see main text) and H-bond geometry optimizations causes a ten-fold increase of the cooperative effect, as both bas- and hyper-HBC contribute to the overall HBC. This is related to Fig. 2 (main text). 


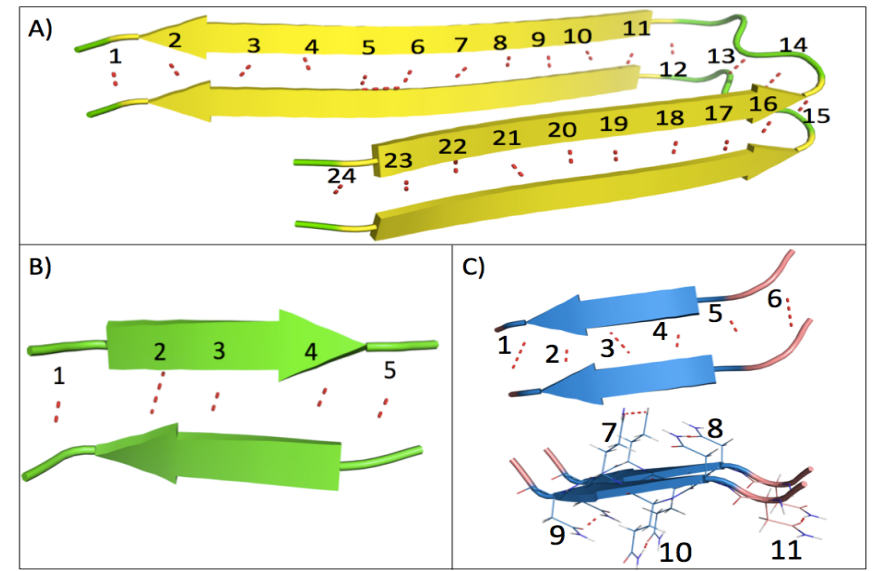

Supplementary Figure 2. Effective number of hydrogen bonds. (A) 24 backbonebackbone (bb-bb) HBs are observed in A $\beta 17-42$, as it is composed of 25 residues: LVFFAEDVGSNKGAIIGLMVGGVVIA. (B) In the MVGGVV segment from A $\beta 35$ 40, $5 \mathrm{HBs}$ of this type are present. (C) GNNQQNY, while composed of just one more residue that MVGGVV and thus, showing $6 \mathrm{bb}-\mathrm{bb}$ hydrogen bonds, is able to form 5 additional side chain-side chain (sc-sc) HBs through its Gln and Asn residues, which sums up to a total of $11 \mathrm{HB}$ interactions. We use the term effective HB number to account for this situation. Note that the polyGly model system (Fig. S1) is identical in the number of bb-bb H-bonds (6) and the parallel register. The only difference is that the five sc-sc interactions are absent as the glutamine $(\mathrm{Q})$ and asparagine $(\mathrm{N})$ residues were mutated to glycines.
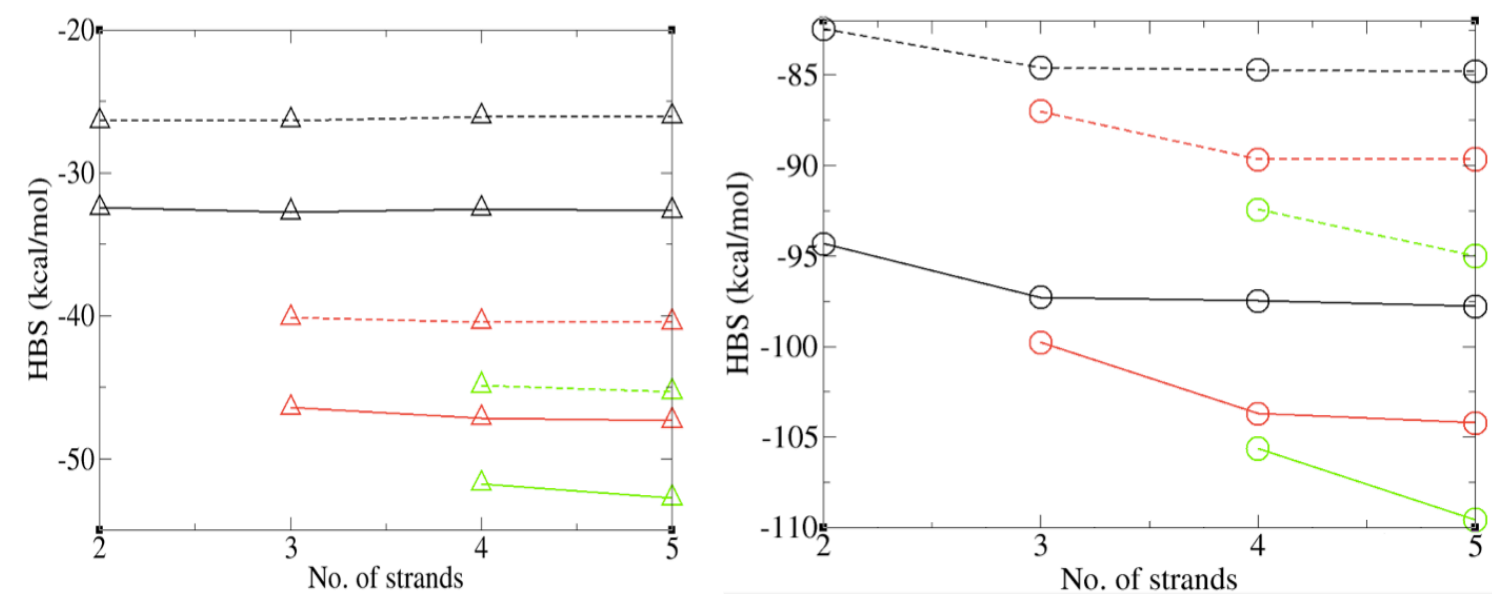

Supplementary Figure 3. Interaction energies with and without van der Waals corrections in MVGGVV (left) and GNNQQNY (right).

The same calculations shown in Fig. 2 in the main text were repeated without the Grimme's correction (dashed lines). As can be seen, the HBC is not altered and inclusion of $\mathrm{vdW}$ interactions results in more stabilizing energies. Note that symbols and color codes are those used in Fig. 2 and both y-axes span 30-35 kcal/mol. 


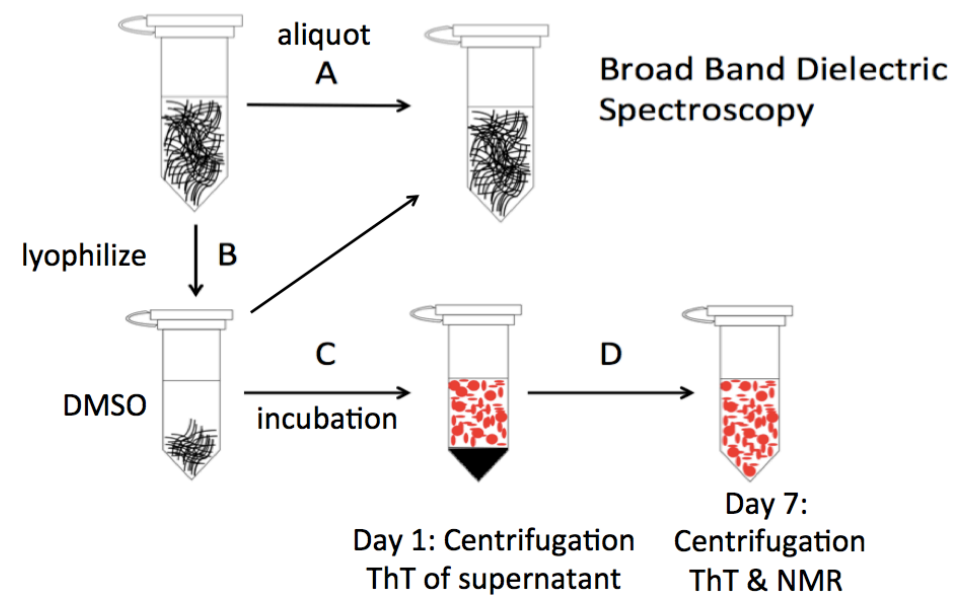

Supplementary Figure 4. Schematic representation of the experimental procedures. Fibrils of $A \beta 17-42$ and GNNQQNY where incubated in water to allow extensive aggregation for at least 21 days (parent samples). (A) $20 \mu \mathrm{L}$ of fibrils from parent samples in water were used for BBDS. Additionally, lyophilized samples were transferred to DMSO-d6 and measured with the same technique. (B) Equal volumes of fibrils from the parent samples were lyophilized and dissolved in DMSO-d6. (C) Samples were incubated for one day and centrifuged to separate fibrils from soluble oligomers and monomers. ThT fluorescence was sharply enhanced by the fibrils; this is good evidence they are amyloid-like (related to Fig. 4A, main text). (D) ThT binding assays were repeated after six days, to test the resistance of the oligomeric species to dissociation by DMSO. NMR revealed dissociation into monomers in the case of polar fibrils, but not in hydrophobic aggregates (related to Fig. 4B-C, main text).

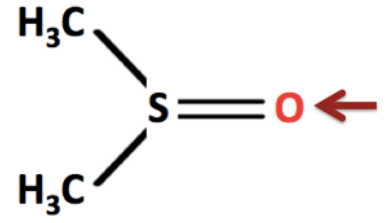

DMSO<smiles></smiles>

DMSO-d6

Supplementary Figure 5. Molecular structures of DMSO and DMSO-d6. Dimethyl sulfoxide was used to solvate the hydrophobic and Gln/Asn-rich oligomers to test the effect of this strong H-bond acceptor. The deuterated version, which is required for the NMR analysis (DMSO-d6), was used in all the experimental measurements in order to perform all the experiments under the same conditions. Note that the color code is that of Fig. 1 in the main text. 


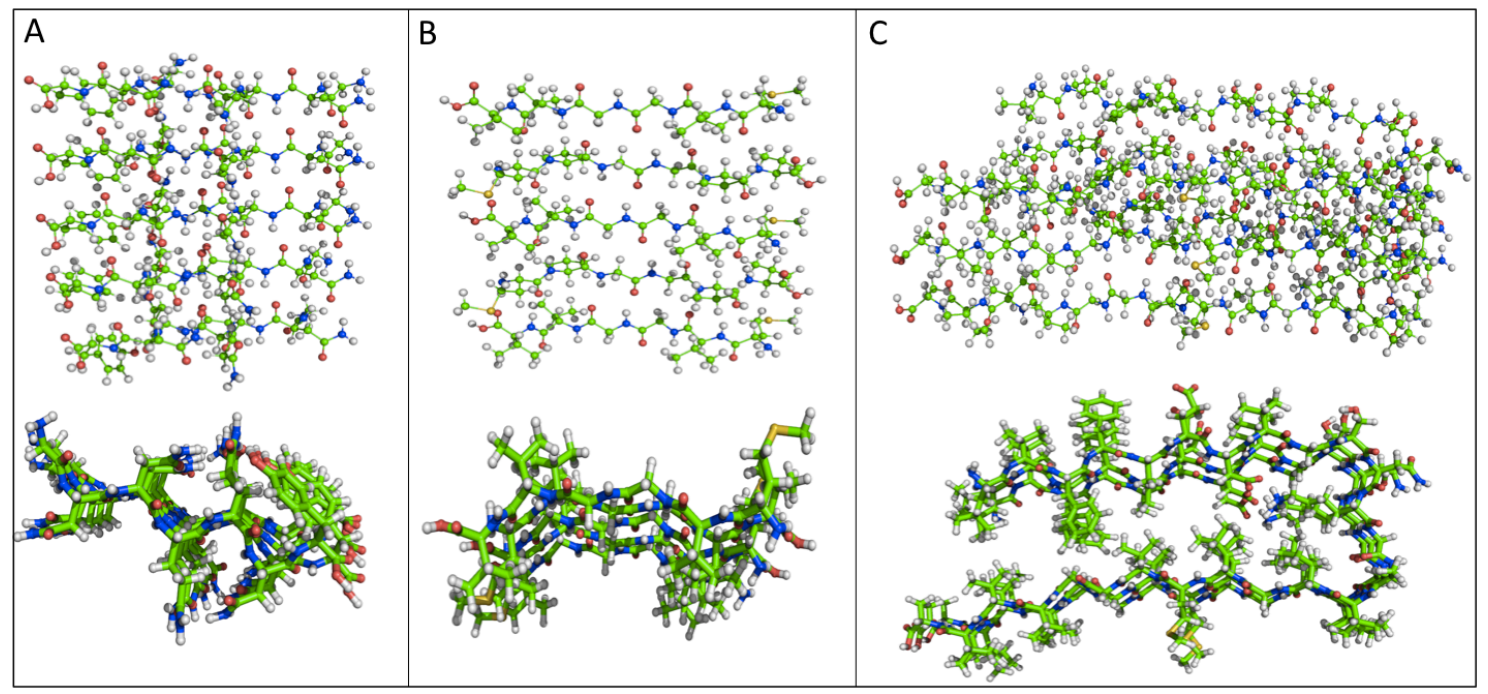

Supplementary Figure 6. Geometries of the peptides used in the calculations and experiments. (A) A pentamer of GNNQQNY. (B) A pentamer of MVGGVV, corresponding to residues $35-40$ of the A $\beta$ peptide. (C) A trimer of the larger hydrophobic segment formed by residues $17-42$ of the $A \beta$ peptide. Due to the vast number of atoms, this trimer was the largest system in which convergence was obtained (see main text). The color code is green for carbon, blue for nitrogen, red for oxygen, white for hydrogen and yellow for sulfur. The all-atom representation is shown as balls in the top figures (side view), and a stick\&balls representation is shown in bottom figures (top view). This figure is related to Fig. 2A, where the schematic representation of the interacting fragments is shown. 


\section{-Supplementary Tables}

Supplementary Table 1. NBO analysis of the HBC. The N lone pair of amide groups from Gln and Asn sidechains, unlike backbone amides, delocalizes over an sp-rich antibonding orbital of the carbonyl group. The stabilization energy provided by these orbitals' overlap can be estimated from second order perturbation theory. Sidechain is abbreviated as $S C$.

\begin{tabular}{|c|c|c|c|}
\hline & $\begin{array}{c}\text { BD*(CO) } \\
\% \text { s-character (C / O) }\end{array}$ & $\begin{array}{c}\text { BD*(CO) } \\
\% \text { p-character }(\mathrm{C} / 0)\end{array}$ & $\begin{array}{c}\mathrm{LP}(\mathrm{N}) \rightarrow \mathrm{BD}(\mathrm{CO}) \\
\Delta \mathrm{E}(\mathrm{kcal} / \mathrm{mol})\end{array}$ \\
\hline Asn2 SC & $30.73 / 41.08$ & $69.17 / 58.58$ & 1.61 \\
\hline Asn3 SC & $30.97 / 38.52$ & $68.93 / 61.45$ & 2.16 \\
\hline Gln4 SC & $31.28 / 41.46$ & $68.63 / 58.20$ & 2.20 \\
\hline Gln5 SC & $30.46 / 41.74$ & $69.44 / 57.94$ & 1.20 \\
\hline Asn6 SC & $29.81 / 39.64$ & $70.09 / 60.02$ & 2.48 \\
\hline
\end{tabular}


Supplementary Table 2. NBO Analysis of the hydrogen bonds. Hydrogen bonds are defined by the electron donation from the oxygen lone pair into an empty antibonding orbital on the $\mathrm{N}-\mathrm{H}$ acceptor. On the basis of the NBO formalism, it can be shown that the oxygen atoms' two lone pairs of electrons are stereoelectronically distinct: whereas one is of $\sim 100 \%$ p-character, the lone pair involved in hydrogen bonding is an s-rich orbital ( $\sim 60 \%$ s-character and $\sim 40 \%$ p-character) [Gray HB Electrons and Chemical Bonding Pub. W. A. Benjamin New York 1965]. We analyzed the HBs in GNNQQNY and MVGGVV on the basis of the optimized wavefunctions at the level of theory used in this work $(\mathrm{M} 06-2 \mathrm{X} / 6-31+\mathrm{G}(\mathrm{d}))$ to ensure that the NBO analysis could be successfully applied to these systems, since to date we are unaware of an NBO study of amyloids fibrils. The slash (/) is used to separate data values for GNNQQNY (left) from MVGGVV (right). Therefore, for the Gln/Asn-rich sequence, there are six values corresponding to six backbone $\mathrm{CONH}$ groups (BB \#1-6), while the five values for the six-residue hydrophobic system are shown on the left side of the slash. Only GNNQQNY is able to form side chain - side chain H-bonds. The last column contains the population of these empty $\mathrm{BD}^{*}(\mathrm{~N}-\mathrm{H})$ orbitals following the same convention (GNNQQNY / MVGGVV).

\begin{tabular}{|c|c|c|c|}
\hline & $\begin{array}{c}\mathrm{LP}(0) \\
\text { \% s-character }\end{array}$ & $\begin{array}{c}\mathrm{LP}(\mathrm{O}) \\
\% \text { p-character }\end{array}$ & $\begin{array}{c}\text { BD*(N-H) } \\
\text { population }\end{array}$ \\
\hline BB \#1 & $56.67 / 59.63$ & $43.28 / 40.33$ & $0.03227 / 0.01681$ \\
\hline BB \#2 & $56.93 / 60.09$ & $43.03 / 40.66$ & $0.03894 / 0.01990$ \\
\hline BB \#3 & $56.57 / 60.46$ & $43.40 / 39.49$ & $0.04313 / 0.01940$ \\
\hline BB \#4 & $57.35 / 59.79$ & $42.61 / 40.17$ & $0.03484 / 0.01714$ \\
\hline BB \#5 & $56.58 / 59.67$ & $43.39 / 40.28$ & $0.03631 / 0.01882$ \\
\hline BB \#6 & $56.89 /-$ & $43.07 /-$ & $0.02749 /-$ \\
\hline SC \#1 & 57.28 & 42.67 & 0.02425 \\
\hline SC \#2 & 57.64 & 42.33 & 0.02303 \\
\hline SC \#3 & 58.22 & 41.74 & 0.02157 \\
\hline SC \#4 & 54.54 & 45.40 & 0.02861 \\
\hline SC \#5 & 57.55 & 42.41 & 0.02766 \\
\hline
\end{tabular}

